Tecnología y

Ciencias $₫$ Agua
2022, Instituto Mexicano de Tecnología del Agua

Open Access bajo la licencia CC BY-NC-SA 4.0 (https://creativecommons.org/licenses/by-nc$\mathrm{sa} / 4.0 /)$

DOI: $10.24850 /$ j-tyca-2022-02-03

Artículos

\title{
Simulación hidrológica de la cuenca del río Laja con el modelo WEAP
}

\section{Hydrological simulation of the Laja River basin with the WEAP model}

Claudia Molina Sánchez ${ }^{1}$ ORCID: https://orcid.org/0000-0001-82909039

Jesús Chávez-Morales² ORCID: https://orcid.org/0000-0002-2894-5853

Oscar Luis Palacios-Vélez³ ORCID: https://orcid.org/0000-0002-79072802

Laura Alicia Ibáñez-Castillo4, ORCID: https://orcid.org/0000-0001-9287$655 X$

${ }^{1}$ Colegio de Postgraduados, Montecillo, Texcoco, Estado de México, México, claudia.molina.san@gmail.com

${ }^{2}$ Colegio de Postgraduados, Montecillo, Texcoco, Estado de México, México, chavezje@colpos.mx

${ }^{3}$ Colegio de Postgraduados, Montecillo, Texcoco, Estado de México, México, opalacio@colpos.mx 
Tecnología y

Ciencias $\stackrel{\Xi}{\varpi}$ gua
2022, Instituto Mexicano de Tecnología del Agua

Open Access bajo la licencia CC BY-NC-SA 4.0 (https://creativecommons.org/licenses/by-nc$\mathrm{sa} / 4.0 /)$

4Universidad Autónoma Chapingo, Postgrado en Ingeniería Agrícola y Uso Integral del Agua, carretera México-Texcoco, Chapingo, Texcoco, Estado de México, México, libacas@gmail.com

Autora para correspondencia: Claudia Molina-Sánchez, claudia.molina.san@gmail.com

\section{Resumen}

La cuenca del río Laja se localiza entre los paralelos $21^{\circ} 32^{\prime} 58^{\prime \prime}, 20^{\circ} 16^{\prime}$ $14^{\prime \prime} \mathrm{N}$ y los meridianos $-100^{\circ} 6^{\prime} 28^{\prime \prime}-101^{\circ} 30^{\prime} 44^{\prime \prime} \mathrm{O}$; en 2014 fue clasificada con grado de presión alto sobre el recurso hídrico (Conagua, 2014); ocupó el segundo lugar como región hidrológica administrativa con mayor densidad de población, y presentó fuertes problemas con oferta y demanda del recurso, lo que motivó llevar a cabo este trabajo. Se realizó una modelación hidrológica en WEAP (Water Evaluation and Planning) con el método de humedad del suelo. El objetivo fue calibrar, validary estimar escurrimientos superficiales, considerando efectos del cambio en precipitación. Se utilizó información de uso y características físicas de suelo, coeficientes de cultivo y datos mensuales promedios de precipitación, temperaturas medias, humedad relativa, velocidad del viento. Los procesos de calibración y validación se efectuaron en cuatro puntos de la cuenca para un periodo de cinco años por mes en las siguientes estaciones hidrométricas: Puente Dolores, Begoña II, Tres Guerras y Pericos; para cada una de ellas se estimó el coeficiente de determinación $\left(r^{2}\right)$ e índice de eficiencia Nash-Sutcliffe (NSE), y se 
Tecnología y

Ciencias $\stackrel{\unlhd}{\unlhd}$ gua
2022, Instituto Mexicano de Tecnología del Agua Open Access bajo la licencia CC BY-NC-SA 4.0 (https://creativecommons.org/licenses/by-nc$\mathrm{sa} / 4.0 /)$

obtuvieron valores de $r^{2}$ de 0.8 a 0.82 , y de NSE de 0.55 a 0.77 en calibración, y $r^{2}$ de 0.65 a 0.86 y NSE de 0.57 a 0.75 en validación. Se generó información climática con WGEN para 10 años, para generar escurrimientos con las proyecciones del escenario RCP8.5 para el periodo 2012-2022. Se concluye que WEAP es un software capaz de simular correctamente la respuesta de la cuenca del río Laja con el método de humedad del suelo. WEAP simuló correctamente los flujos medidos en la estación de medición con un error absoluto medio inferior al $10 \%$.

Palabras clave: modelación hidrológica, WEAP, calibración, validación, escurrimientos, río Laja.

\section{Abstract}

The Laja River basin is located between parallels $21^{\circ} 32^{\prime} 58^{\prime \prime}, 20^{\circ} 16^{\prime} 14^{\prime \prime}$ $\mathrm{N}$, and meridians - $100^{\circ} 6^{\prime} 28^{\prime \prime}-101^{\circ} 30^{\prime} 44^{\prime \prime} \mathrm{W}$. In 2014, the basin was classified with a high degree of pressure on the water resource (Conagua, 2014). It also ranked second as an administrative hydrological region with the highest population density, and it presents strong problems with supply and demand. All the previous reasons motivated this work. The objective was to calibrate, validate and estimate runoff surface, considering the effects of changes in precipitation, Therefore, hydrological modeling was performed in WEAP (Water Evaluation and Planning) using the soil moisture method. The information used was on soil use and physical characteristics; crop coefficients; average monthly data on precipitation; average temperatures; relative humidity; and wind speed. The calibration and validation processes were carried out at four points in 
Tecnología y

Ciencias $\stackrel{\unlhd}{\unlhd}$ gua
2022, Instituto Mexicano de Tecnología del Agua

Open Access bajo la licencia CC BY-NC-SA 4.0 (https://creativecommons.org/licenses/by-nc$\mathrm{sa} / 4.0 /)$

the basin, for five years on a monthly period. The hydrometric stations were Puente Dolores, Begoña II, Tres Guerras and Pericos. For each station, the coefficient of determination $\left(r^{2}\right)$ and Nash-Sutcliffe efficiency index (NSE) were estimated. The values obtained in the calibration phase were an $r^{2}$ of 0.8 to 0.82 and NSE from 0.55 to 0.77 . In the validation phase, the values for $r^{2}$ range from 0.65 to 0.86 and NSE from 0.57 to 0.75. Climate information was generated with WGEN for 10 years, to generate runoff scenarios, with the RCP8.5 projections, for the period 2012-2022. In conclusion, WEAP is a software capable of correctly simulating, with the soil moisture method, the response of the Laja River basin. WEAP correctly simulated flows measured in the gauging station with an average absolute error of less than $10 \%$.

Keywords: hydrological modeling, WEAP, calibration, validation, runoff, Laja River.

Recibido: 24/01/2019

Aceptado: 25/01/2021

\section{Introducción}


Tecnología y

Ciencias $\stackrel{\unlhd}{\unlhd}$ gua
2022, Instituto Mexicano de Tecnología del Agua

Open Access bajo la licencia CC BY-NC-SA 4.0 (https://creativecommons.org/licenses/by-nc$\mathrm{sa} / 4.0 /)$

El agua es un precioso recurso natural vital para la vida, el desarrollo y el medio ambiente. Puede ser una cuestión de vida y muerte, según cómo ocurra y cómo se administre (Hamlat, Errih, \& Guidoum, 2013). Los cambios en la temperatura y la precipitación afectan los procesos hidrológicos, y la disponibilidad de agua para las áreas agrícolas, la energía hidroeléctrica, los sectores industriales y la población. El cambio climático acelerará el ciclo hidrológico con una tendencia creciente en la temperatura y evapotranspiración, y un cambio en la precipitación. El cambio en la intensidad, distribución y frecuencia de la precipitación afectará el flujo superficial (Rochdane, Reichert, Messouli, Babqiqi, \& Khebiza, 2012).

La modelación hidrológica se ha convertido en un componente indispensable en investigación y manejo del recurso hídrico, ya que los modelos hidrológicos ayudan a comprender la situación actual y pasada de éstos en una cuenca, además de proporcionar una manera de explorar las implicaciones de las decisiones de gestión y cambios impuestos como el cambio climático (Johnston \& Smakhtin, 2014).

El modelo WEAP (Water Evaluation and Planning) del Instituto del Medio Ambiente de Estocolmo (SEI) (por sus siglas en inglés Stockholm Environment Institute) (SEI, 2015) se utiliza en el análisis de la disponibilidad y demanda del agua, proporcionando un marco para evaluar demandas sectoriales, medidas de conservación del agua, prioridades en asignación, operación de acuíferos, y costos de proyectos y beneficios (Yates, Sieber, Purkey, Huber-Lee, \& Galbraith, 2005b). Es particularmente útil para comparar escenarios de cambio hidrológico 
Tecnología y

Ciencias $\stackrel{\unlhd}{\unlhd}$ gua
2022, Instituto Mexicano de Tecnología del Agua Open Access bajo la licencia CC BY-NC-SA 4.0 (https://creativecommons.org/licenses/by-nc$\mathrm{sa} / 4.0 /)$

(Höllermann, Giertz, \& Diekkrüger, 2010; Harma, Johnson, \& Cohen, 2012) y se ha utilizado para evaluar opciones de adaptación en el nivel urbano (Bonelli, Vicuña, Meza, Gironás, \& Barton, 2014; Yates, Miller, Wilby, \& Kaatz, 2015a).

Los escenarios futuros que se pueden analizar son de diferentes tipos: de crecimiento o cambio en la población, de uso de suelo, de clima. Los escenarios de clima constituyen uno de los aspectos de mayor uso en un modelo WEAP. Existen diferentes formas de implementarproyecciones climáticas dentro de un modelo, como incorporación de cambios o variaciones de temperatura y/o precipitación con base en proyecciones de modelos climáticos, y uso de datos escalados a la región (CCG, 2009; SEI, 2015).

El modelo WEAP se ha utilizado en diferentes partes del mundo: el Instituto Coreano de Tecnología de la construcción adoptó WEAP como una herramienta para ayudar en su planificación de suministro de agua a largo plazo, modelado de la calidad del agua superficial y análisis de beneficios-costos (Choi, Kim, \& Lee, 2012); el plan de agua de California, mediante la aplicación de WEAP, proporcionó un marco para que los administradores del agua, los legisladores y el público consideren opciones, y tomen decisiones con respecto al futuro del agua en Califomia (Savage et al., 2004); en Guatemala se utilizó para estimar la vulnerabilidad al cambio climático (Magaña, 2005).

En México se ha aplicado WEAP en diferentes lugares. En las cuencas del río Guayalejo-Tamesí, Tamaulipas, Sánchez-Torres-Esqueda, Ospina-Noreña, Gay-García y Conde (2011) abordaron el impacto del 
Tecnología y

Ciencias $₫$ Agua
2022, Instituto Mexicano de Tecnología del Agua

Open Access bajo la licencia CC BY-NC-SA 4.0 (https://creativecommons.org/licenses/by-nc$\mathrm{sa} / 4.0 /)$

cambio climático en la variabilidad de la disponibilidad de agua mediante el desarrollo de escenarios a corto, mediano y largo plazos. En la cuenca del Río Grande/Bravo, Sandoval-Solis y McKinney (2009) describieron el proceso de modelación colaborativo para construir una planificación del recurso hídrico, definir y evaluar escenarios. En la cuenca del río Sextín o del Oro, ubicada al centro-noreste de Durango, México, Esquivel, Nevárez, Velásquez, Sánchez y Bueno (2017) evaluaron el impacto de los patrones climáticos sobre el escurrimiento de la cuenca.

En la república mexicana, grandes ríos conforman un gran número de cuencas habitadas por grupos poblacionales dispersos que ejercen una fuerte presión sobre los frágiles recursos, especialmente en el hídrico. La cuenca del río Laja en Guanajuato presenta estas características en su parte alta, lo que repercute en sus partes media y baja (Torres-Benites, Mejía-Sáenz, Cortés-Becerra, Palacios-Vélez, \& Exebio-García, 2005). En la cuenca del río Laja, la planificación de los recursos hídricos es una necesidad inmediata, ya que por años el manejo del recurso hídrico ha estado supeditado a las necesidades de abastecimiento del agua sin previo análisis del comportamiento hidrológico de la cuenca. Eso ha originado problemas en la disponibilidad del recurso, sobre todo en su parte baja, con sectores severamente afectados, como es el agrícola (Palacios-Vélez, \& López-López, 2004). Este trabajo tuvo el objetivo de realizar simulación hidrológica con el "Soil Moisture Method" (método de la humedad del suelo) para calibrar, validar y estimar escurrimientos superficiales, tomando en cuenta efectos del cambio climático con las proyecciones del escenario RCP8.5. 
Tecnología y

Ciencias $\stackrel{\mho}{\varpi}$ Aua
2022, Instituto Mexicano de Tecnología del Agua

Open Access bajo la licencia CC BY-NC-SA 4.0 (https://creativecommons.org/licenses/by-nc$\mathrm{sa} / 4.0 /)$

\section{Materiales y métodos}

\section{Cuenca de estudio}

La cuenca del río Laja (Figura 1) se localiza entre los paralelos $20^{\circ} 16^{\prime}$ $14^{\prime \prime}, 21^{\circ} 32^{\prime} 58^{\prime \prime} \mathrm{N}$, y entre los meridianos $-100^{\circ} 06^{\prime} 28^{\prime \prime},-101^{\circ} 30^{\prime} 44^{\prime \prime}$ O. Pertenece a la región hidrológica número 12, denominada "Lerma Santiago"; con fines de gestión del recurso hídrico pertenece a la Región Hidrológico-Administrativa VIII "Lerma Santiago Pacifico". De acuerdo con la clasificación de Campos (1998), la cuenca se clasifica como muy grande (11 $912 \mathrm{~km}^{2}$ ). Dentro de la superficie que cubre la cuenca del río Laja se encuentran 32 municipios, sin embargo, nueve son los que representan el mayor porcentaje: Dolores Hidalgo (14 \%), San Miguel de Allende (13\%), San Felipe (9 \%), San Luis de la Paz (8 \%), San Diego de la Unión (7\%), el Marqués (6\%), Querétaro (6\%), Celaya (5\%) y Comonfort ( $5 \%$ ). El río Laja es tributario del río Lerma. Tiene su origen en el cerro de San Juan con el nombre de río del Nuevo Valle de Moreno, 
Tecnología y

Ciencias $\stackrel{\unlhd}{\unlhd}$ gua
2022, Instituto Mexicano de Tecnología del Agua

Open Access bajo la licencia CC BY-NC-SA 4.0 (https://creativecommons.org/licenses/by-nc$\mathrm{sa} / 4.0 / /)$

hasta donde cruza con la estación del ferrocarril Obregón, Guanajuato, cuando cambia su nombre al de río Laja (DOF, 2003).

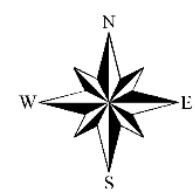

$102^{\circ} 0$ O"W

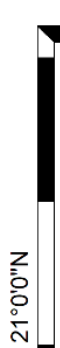

$101^{\circ} 0^{\prime} 0^{\prime \prime} \mathrm{W}$

$100^{\circ} 0^{\prime} 0 " W$

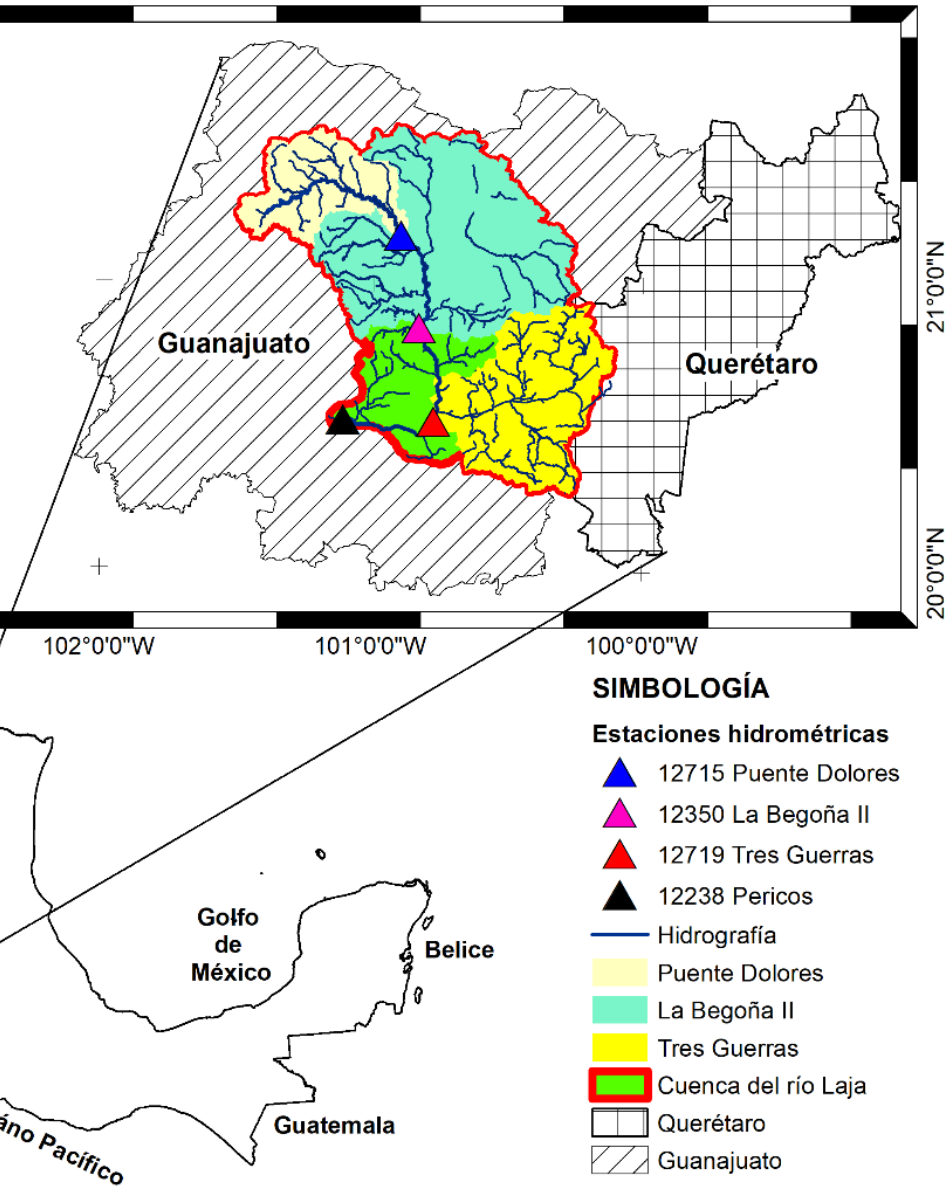

Figura 1. Ubicación de la zona de estudio. Elaboración propia con información de Conagua (2014) e INEGI (2013).

\section{Clima}


Se utilizaron variables climáticas mensuales de series históricas de precipitación y temperatura media mensual registradas en 44 estaciones climatológicas convencionales (EMCs) distribuidas en toda la cuenca (Figura 2) (SMN, 2009). Los valores de la humedad relativa y velocidad del viento se obtuvieron de la red de estaciones meteorológicas automáticas (EMAs) del Instituto Nacional de Investigaciones Forestales, Agrícolas y Pecuarias (INIFAP, 2016). La cobertura de nubes fue tomada de forma empírica. Toda la información climática indicada es requerida por el WEAP para la construcción del modelo y se ingresó cada mes. 
Tecnología y

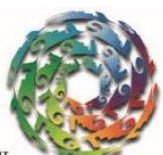

Ciencias $\stackrel{\Im}{\approx}$ gua
2022, Instituto Mexicano de Tecnología del Agua

Open Access bajo la licencia CC BY-NC-SA 4.0 (https://creativecommons.org/licenses/by-nc$\mathrm{sa} / 4.0 /)$

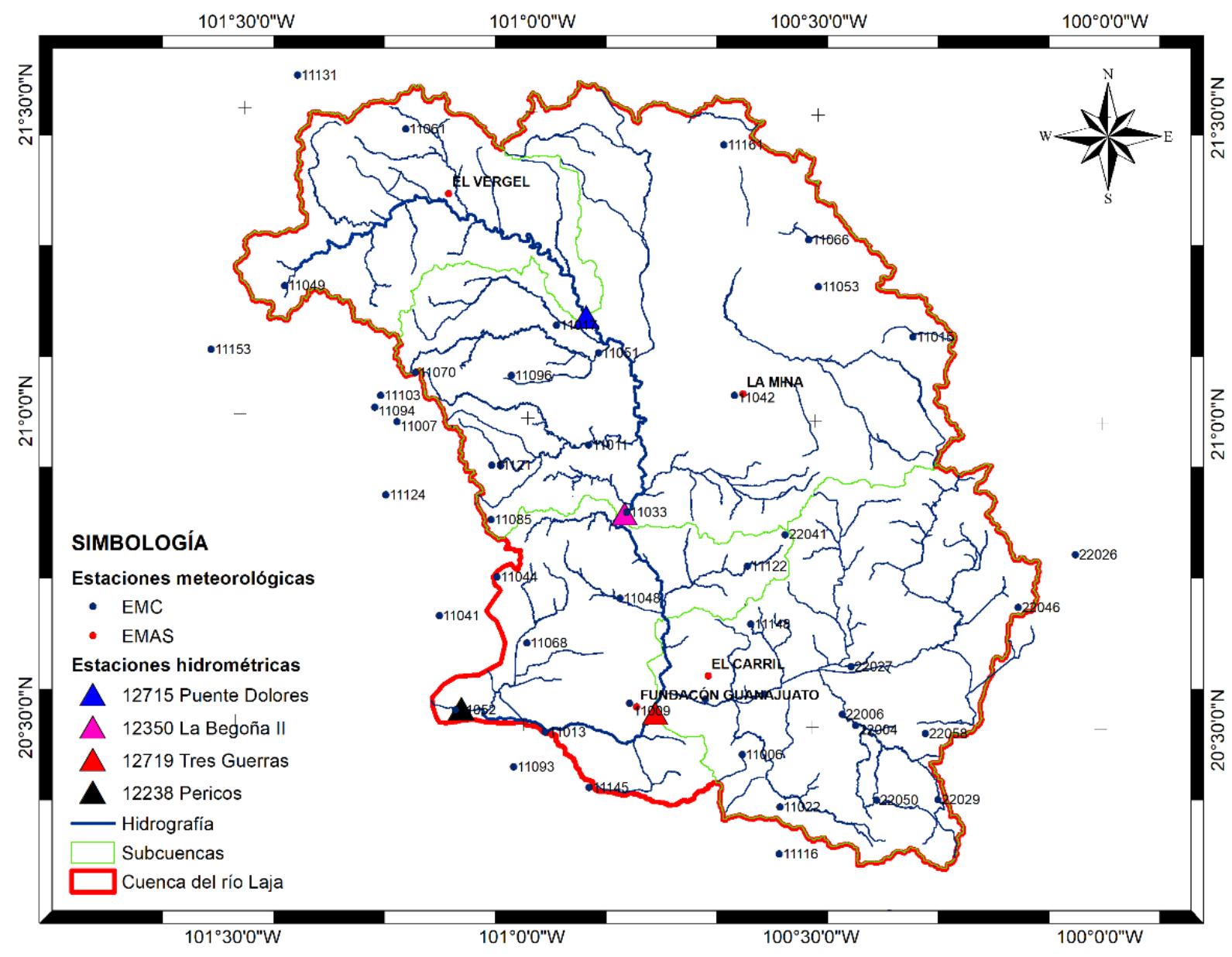

Figura 2. Estaciones climatológicas. Elaboración propia con información del SMN (2009).

\section{Hidrometría}


Tecnología y

Ciencias Agua
2022, Instituto Mexicano de Tecnología del Agua Open Access bajo la licencia CC BY-NC-SA 4.0 (https://creativecommons.org/licenses/by-nc$\mathrm{sa} / 4.0 /)$

Se delimitaron tres subcuencas y la cuenca del río Laja de acuerdo con cuatro estaciones hidrométricas convencionales (EHCs) existentes. La información hidrométrica se obtuvo del Banco Nacional de Aguas Superficiales (Conagua \& IMTA, 2008); los datos utilizados corresponden a los caudales medios mensuales históricos medidos en las cuatro EHCs existentes en la cuenca (iError! No se encuentra el origen de la referencia.). Para calibración y validación se utilizó información de flujos mensuales de cinco años.

Tabla 1. Estaciones hidrométricas en la cuenca (Conagua \& IMTA, 2008).

\begin{tabular}{|l|l|c|c|c|}
\hline Clave & Nombre de EHC & Área drenada $\mathbf{( k m}^{\mathbf{2}} \mathbf{)}$ & Long W & Lat. N \\
\hline 12715 & Puente Dolores & 1711 & -100.90 & 21.17 \\
\hline 12350 & La Begoña II & 4984 & -100.83 & 20.85 \\
\hline 12719 & Tres Guerras & 5849 & -100.77 & 20.52 \\
\hline 12238 & Pericos & 9651 & -101.11 & 20.53 \\
\hline
\end{tabular}

De acuerdo con el método de humedad del suelo que se utilizó para la modelación hidrológica con WEAP se requieren parámetros hidrológicos y climáticos detallados. Los parámetros utilizados fueron coeficientes de cultivo (Kc) asignados con base en Allen, Pereira, Raes y Smith (2006), tomando en cuenta la vegetación y el patrón de cultivos del DR 085 "La Begoña". La capacidad de almacenamiento de agua en la zona de raíces 
Tecnología y

Ciencias $\stackrel{\Xi}{\unlhd}$ gua
2022, Instituto Mexicano de Tecnología del Agua Open Access bajo la licencia CC BY-NC-SA 4.0 (https://creativecommons.org/licenses/by-nc$\mathrm{sa} / 4.0 /)$

(Sw) varía de acuerdo con los tipos de cobertura del suelo, para la cuenca del río laja se propusieron los valores capacidad de almacenamiento de agua en zona profunda ( $D w)$; factor de resistencia al escurrimiento $(R R F)$; conductividad en zona de raíces ( $k s)$; conductividad en zona profunda $(k d)$; dirección preferencial de flujo $(f)$; humedad inicial en zona de raíces (Z1), y humedad inicial en zona profunda (Z2). Los parámetros se ingresaron en porcentaje y valor absoluto. Los ajustes realizados en dichas variables se efectúan considerando los rangos establecidos por el mismo modelo y de otros esquemas hidrológicos similares a la cuenca de estudio (Flores-López, Galaitsi, Escobar, \& Pukey, 2016).

Los valores de los parámetros del modelo WEAP para la cuenca del río Laja se definieron con base en valores de parámetros del método de humedad del suelo de otras aplicaciones realizadas en el continente americano (Amato, McKinney, Ingol-Blanco, \& Teasley, 2006; FloresLópez et al., 2016; Febrillet-Huertas, José-Clases, Bello, \& Chalas, 2014) (Tabla 2).

Tabla 2. Parámetros utilizados en el modelo hidrológico de la cuenca del río Laja.

\begin{tabular}{|l|c|c|c|c|c|c|c|c|}
\hline CLASE & $K c$ & $\begin{array}{c}\text { Sw } \\
(\mathbf{m m})\end{array}$ & $\begin{array}{c}\text { Dw } \\
(\mathbf{m m})\end{array}$ & $\boldsymbol{R} \boldsymbol{k F}$ & $\begin{array}{c}\text { kd } \\
(\mathbf{m m} / \\
\mathbf{m e s})\end{array}$ & $\boldsymbol{f}$ & $\mathbf{z 1} \%$ & $\mathbf{z 2} \%$ \\
\hline Agricultura & 1.2 & 330 & 330 & 2 & 200 & 0.4 & 30 & 30 \\
\hline Bosque & 0.8 & 400 & 400 & 4 & 200 & 0.4 & 30 & 30 \\
\hline
\end{tabular}


Tecnología y

Ciencias ฐAgua
2022, Instituto Mexicano de Tecnología del Agua Open Access bajo la licencia CC BY-NC-SA 4.0 (https://creativecommons.org/licenses/by-nc$\mathrm{sa} / 4.0 /)$

\begin{tabular}{|l|c|c|c|c|c|c|c|c|}
\hline Cuerpo de agua & 0.7 & 100 & 100 & 1 & 200 & 0.4 & 30 & 30 \\
\hline Matorral & 0.8 & 100 & 100 & 2 & 200 & 0.4 & 30 & 30 \\
\hline Pastizal & 0.6 & 330 & 330 & 3 & 200 & 0.4 & 30 & 30 \\
\hline Selva & 0.7 & 400 & 400 & 4 & 200 & 0.4 & 30 & 30 \\
\hline Zona urbana & 0.7 & 100 & 100 & 1 & 200 & 0.4 & 30 & 30 \\
\hline
\end{tabular}

\section{Uso de suelo y vegetación}

La información correspondiente a coberturas para cada una de las subcuencas se obtuvo de la capa vectorial serie IV de uso de suelo y vegetación (INEGI, 2010) escala 1:250 000. La cuenca del río Laja presenta ocho clases de uso de suelo: agricultura (51.2 \%), pastizal (21.25\%), bosque (11.9\%), selva (6.23\%), matorral (6.0\%), zona urbana $(2.3 \%)$, suelo desnudo $(0.7 \%)$ y cuerpos de agua $(0.5 \%)$.

\section{Método de humedad del suelo}


Tecnología y

Ciencias $₫$ Agua
2022, Instituto Mexicano de Tecnología del Agua

Open Access bajo la licencia CC BY-NC-SA 4.0 (https://creativecommons.org/licenses/by-nc$\mathrm{sa} / 4.0 /)$

El método de humedad del suelo es el más complejo de los métodos modelados en WEAP; se basa en funciones empíricas que describen el comportamiento de la evapotranspiración, el escurrimiento superficial, la infiltración, el flujo base y la filtración profunda para una cuenca (SEI, 2015). El modelo considera el movimiento del agua a través de dos capas verticales de suelo (Figura 3). La primera capa representa el agua retenida cerca de la superficie, que está disponible para las raíces de las plantas; la segunda capa es más profunda y el agua de ésta se puede transmitir como flujo base o recarga de agua subterránea. Los principales parámetros de este modelo incluyen la capacidad de retención de agua para ambas capas, así como la conductividad hidráulica saturada de cada capa (Yates et al., 2005b; Yates, Sieber, Purkey, Huber-Lee, \& Galbraith, 2005c; SEI, 2015). El modelo calcula el balance hídrico debido a las entradas, salidas y cambios de almacenamiento en cada capa para cada subcuenca de una cuenca. 
Tecnología y

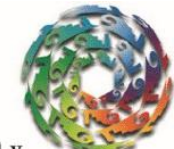

Ciencias

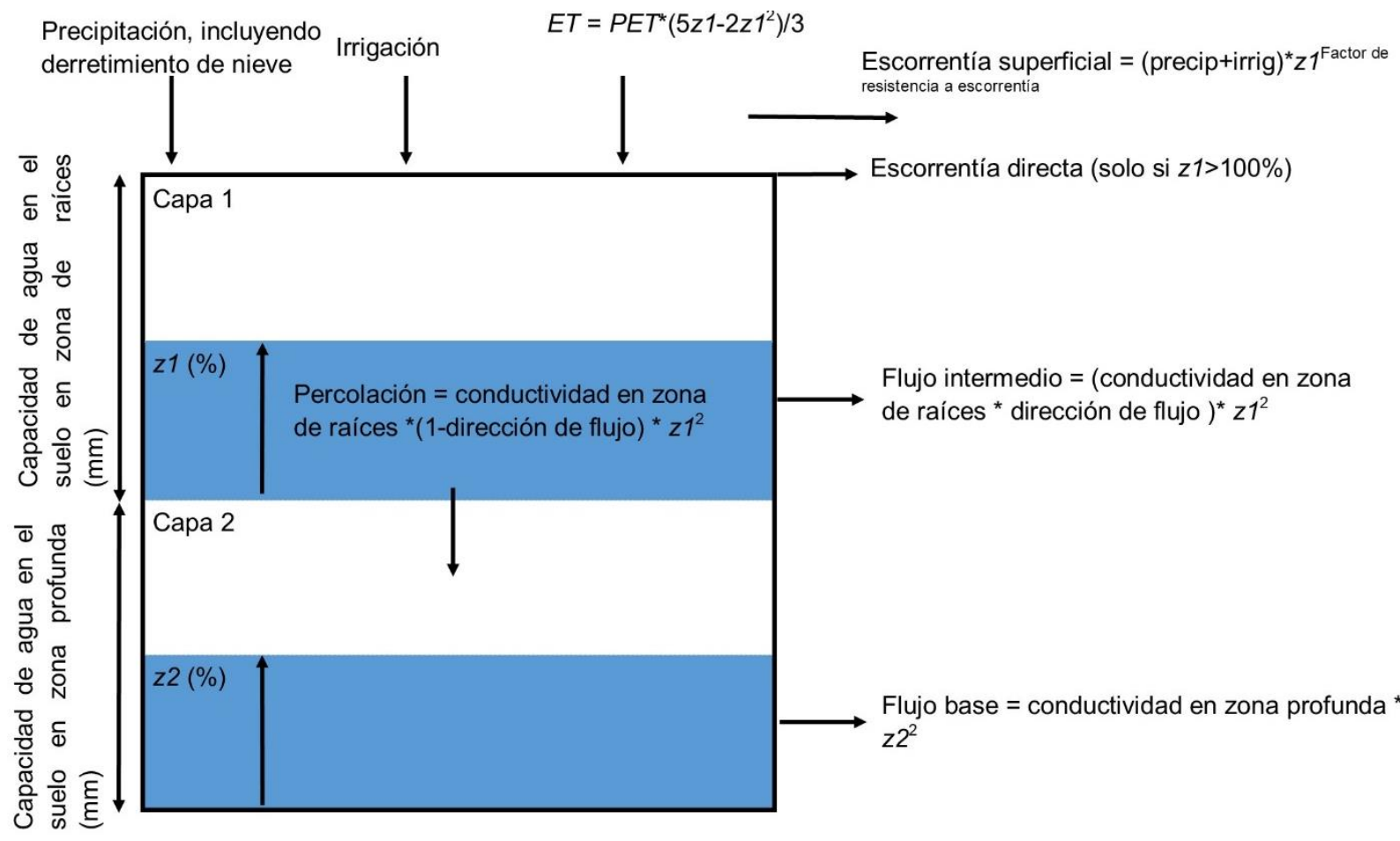

$E T$ : evapotranspiración; PET: evapotranspiración potencial; z1: nivel de humedad inicial en zona de raíces; $z 2$ : nivel de humedad inicial en zona profunda.

Figura 3. Elementos hidrológicos modelados en WEAP (CCG, 2009).

El cambio de humedad del suelo en la primera capa está dado por la precipitación efectiva menos la evapotranspiración de referencia, escorrentía superficial, infiltración y percolación. Una unidad de cuenca se puede dividir en $N$ áreas fraccionarias por subcuencas que representan diferentes usos de la tierra/tipos de suelo; el balance hídrico se calcula para cada área fraccional $j$ de $N$. Se supone que el clima es uniforme en cada subcuenca y se da el balance hídrico. Se plantea como (SEI, 2015): 
Tecnología y Ciencias $₫$ Agua
2022, Instituto Mexicano de Tecnología del Agua

Open Access bajo la licencia CC BY-NC-SA 4.0 (https://creativecommons.org/licenses/by-nc$\mathrm{sa} / 4.0 /$ )

$R d_{j} \frac{d z_{1 j}}{d t}=P e(t)-P E T(t) k_{c, j}(t)\left(\frac{5 z_{1, j}-2 z_{1}{ }^{2}, j}{3}\right)-P e(t) z_{1_{j}}{ }^{\mathrm{RRF}}-f_{j} k_{s, j} z_{1}{ }^{2}{ }_{j}-\left(1-f_{j}\right) k_{s, j} z_{1}{ }^{2}{ }_{j}$

Donde:

$R d_{j} \frac{d z_{1_{j}}}{d t}=$ cambio en humedad del suelo en la capa 1 del área $j(\mathrm{~mm})$.

$P e(t)=$ precipitación efectiva en el tiempo $t(\mathrm{~mm})$.

$\operatorname{PET}(t)=$ evapotranspiración potencial para el área $j$ en el tiempo $t$.

$k_{c_{j}}=$ coeficiente de cultivo para el área $j$.

$z_{1, j}=$ almacenamiento relativo dado como una fracción del almacenamiento efectivo total de la capa de zona de raíces para el área $j$.

$R R F_{j}=$ factor de resistencia al escurrimiento (0-1000). El escurrimiento decrece con los valores más altos.

$\operatorname{Pe}(t) z_{1_{j}}{ }^{R F_{j}}=$ escorrentía superficial.

$f_{j} k_{s, j} z_{1}^{2}{ }_{j}=$ infiltración de la primera capa para el área $j$.

$\left(1-f_{j}\right) k_{s, j} z_{1}^{2}{ }_{j}=$ percolación.

$f_{j}=$ coeficiente relacionado con el suelo, el tipo de cobertura de la tierra y la topografía que definen la dirección de flujo en la capa 1 (0-1).

$k_{s, j}=$ estimación de la conductividad saturada en zona de raíz $\left(\mathrm{mmh}^{-1}\right)$.

$(t)=$ tiempo 
Tecnología y

Ciencias $\stackrel{\unlhd}{\unlhd}$ gua
2022, Instituto Mexicano de Tecnología del Agua

Open Access bajo la licencia CC BY-NC-SA 4.0 (https://creativecommons.org/licenses/by-nc$\mathrm{sa} / 4.0 /)$

El cambio en el almacenamiento de la segunda capa se calcula con:

$S_{\text {máx }} \frac{d z_{2}}{d t}=\left[\sum_{j=1}^{N}\left(1-f_{j}\right) k_{s, j} z_{1}{ }^{2}{ }_{j}\right]-k_{s, 2} z_{2}{ }^{2}$

Donde:

$S_{\text {máx }}=$ la percolación profunda del almacenamiento en la capa superior.

$k_{s_{2}}=$ la conductividad hidráulica saturada de la capa de almacenamiento inferior para el área $j$ y se da como un valor único para la cuenca(mm/tiempo).

$f_{j}=$ es un coeficiente relacionado con el suelo, el tipo de cobertura de la tierra y la topografía que definen la dirección de flujo en la capa $2(0-1)$. $z_{2, j}=$ es el almacenamiento relativo dado como un porcentaje del almacenamiento efectivo de la capa inferior del suelo para el área $j$.

\section{Metodología}

\section{Modelación hidrológica}


La modelación hidrológica se inicia con la delimitación de la cuenca y subcuencas, empleando un modelo digital de elevación MDE con una resolución de $15 \mathrm{~m}$, escala 1:50 000 (INEGI, 2013). La metodología para la modelación hidrológica en WEAP se realizó siguiendo los pasos mostrados en el diagrama de la Figura 4. 
Tecnología y

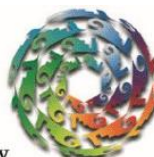

Ciencias 공a
2022, Instituto Mexicano de Tecnología del Agua

Open Access bajo la licencia CC BY-NC-SA 4.0 (https://creativecommons.org/licenses/by-nc$\mathrm{sa} / 4.0 /)$

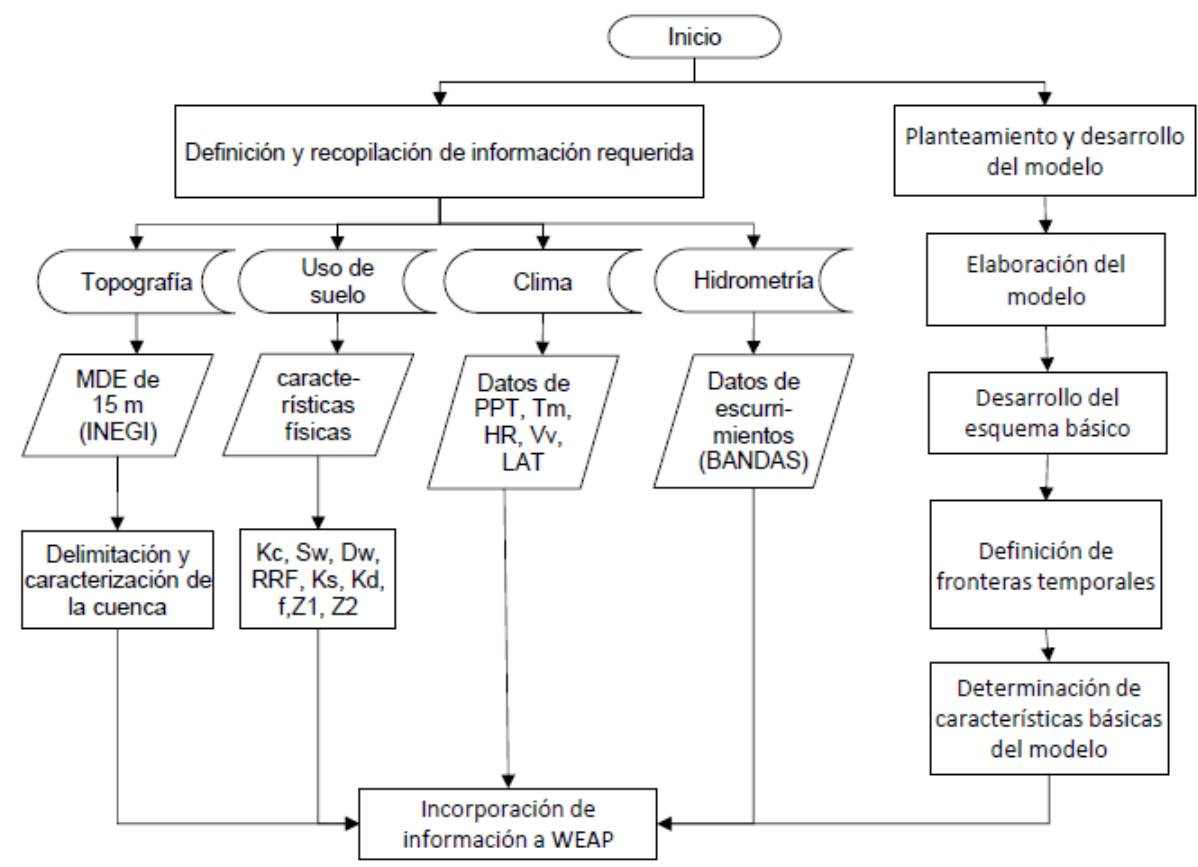

Kc: coeficiente de cultivo

Sw: capacidad de almacenamiento en la zona radical

Dw: capacidad de almacenamiento de agua en la zona profunda

RRF: factor de resistencia al

escurrimiento

Ks: conductividad en zona de raices

Kd: conductividad en zona profunda

f: dirección preferencial del flujo (0-1)

Z1: humedad inicial en zona de raices

$\mathrm{Z2}$ : humedad inicial en zona profunda

PPT: precipitación ( $\mathrm{mm}$ )

Tm: temperatura media $\left({ }^{\circ} \mathrm{C}\right)$

HR: humedad relativa $(\%)$

Vv: velocidad del viento

Lat: latitud

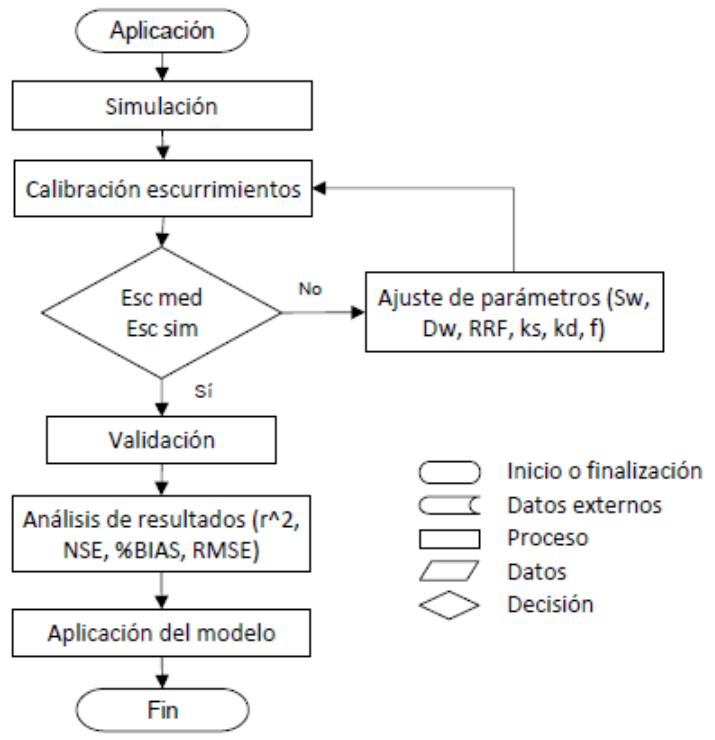

Figura 4. Diagrama de la metodología para calibrar y validar el modelo WEAP en la cuenca del río Laja, Guanajuato, México. 


\section{Construcción del modelo hidrológico de la cuenca del río Laja en WEAP}

El proceso de modelación se elaboró con la interacción de los elementos naturales y antrópicos, que a su vez se componen de parámetros. Definidos los parámetros e información requerida por el modelo se procedió a ingresarlos en la interfase del modelo WEAP. Se hizo la primera simulación con los parámetros ingresados y se observó que los resultados producidos por el modelo reprodujeran en cada subcuenca y cuenca los picos de precipitación observados en las EMCs, y de los flujos observados en las EHCs. Una vez desarrollado y obtenido un resultado aceptable se procedió a su calibración.

\section{Calibración}


Tecnología y

Ciencias $₫$ Agua
2022, Instituto Mexicano de Tecnología del Agua Open Access bajo la licencia CC BY-NC-SA 4.0 (https://creativecommons.org/licenses/by-nc$\mathrm{sa} / 4.0 /)$

El proceso de calibración consiste en ajustar los parámetros del modelo variándolos hasta que los valores a la salida del modelo se ajustan a los datos observados reales (Cabrera, 2012).

La calibración se llevó a cabo mediante la comparación de los escurrimientos medidos a la salida de cada subcuenca versus escurrimientos simulados por WEAP para el periodo 1989-1993, periodo elegido al ser el más reciente que presentaba un registro continuo de datos en todas las estaciones hidrométricas.

\section{Validación}

Refsgaard y Knudsen (1996) definen la validación de un modelo como "el proceso de demostración de que el modelo es capaz de hacer predicciones en un lugar específico determinado para periodos fuera del periodo de calibración". En este estudio, la validación se realizó con información hidrométrica de un periodo de cinco años (2001-2005 para las EHC Begoña II, Tres guerras y Pericos; y 1984-1988 para la EHC Puente Dolores), correspondiente a los cinco años más recientes de la información existente. Para medir la eficacia predictiva del modelo en la calibración y validación se usaron indicadores utilizados frecuentemente en modelación hidrológica: coeficiente de determinación $\left(r^{2}\right)$, error medio 
Tecnología y

Ciencias $\stackrel{\unlhd}{\unlhd}$ gua
2022, Instituto Mexicano de Tecnología del Agua

Open Access bajo la licencia CC BY-NC-SA 4.0 (https://creativecommons.org/licenses/by-nc$\mathrm{sa} / 4.0 /)$

absoluto (MAE), porcentaje de sesgo (\%BIAS) y coeficiente de eficiencia de Nash-Sutcliffe (NSE) (Ahmed, 2012).

\section{Resultados y discusión}

Se formuló, calibró y validó el modelo hidrológico en las subcuencas y la cuenca del río Laja con la información de precipitación de las EMCs y de cuatro estaciones hidrométricas convencionales de aforo. Los parámetros que presentaron mayor sensibilidad y que se ajustaron dentro del modelo correspondieron a la capacidad de retención de humedad del suelo (SWC), conductividad en zona de raíces (RZC), factor de resistencia al flujo (RRF) y dirección del flujo preferencial (PFD); todos ellos son parámetros que caracterizan al suelo y sus horizontes, información escasa en nuestro país.

El resultado obtenido en la modelación se ve afectado por la calidad de los datos que lo sustentan.

El error obtenido entre flujos simulados versus medidos es menor del $10 \%$ en promedio. 
Tecnología y

Ciencias $\stackrel{\unlhd}{\unlhd}$ gua
2022, Instituto Mexicano de Tecnología del Agua

Open Access bajo la licencia CC BY-NC-SA 4.0 (https://creativecommons.org/licenses/by-nc$\mathrm{sa} / 4.0 /)$

\section{Calibración}

Las pruebas de bondad de ajuste aplicadas durante la calibración a los resultados del modelo hidrológico del río Laja mostraron buena correlación entre los caudales simulados por el modelo WEAP y observados en las EHCs analizadas, Puente Dolores, La Begoña II, Tres Guerras y Pericos (Figura 5). Los índices estadísticos de Nash-Sutcliffe = $0.55,0.6,0.77$ y 0.74, y $r^{2}$ de $0.82,0.88,0.81,0.8$, respectivamente, muestran dicha correlación (Tabla 3).

Tabla 3. Medidas de bondad de ajuste para la calibración en las subcuencas y cuenca del río Laja.

\begin{tabular}{|l|c|c|c|c|c|c|c|c|}
\hline \multirow{2}{*}{ Prueba } & \multicolumn{2}{|c|}{$\begin{array}{c}\text { EHC Puente } \\
\text { dolores }\end{array}$} & \multicolumn{2}{|c|}{ EHC Begoña II } & \multicolumn{2}{c|}{$\begin{array}{c}\text { EHC Tres } \\
\text { Guerras }\end{array}$} & \multicolumn{2}{c|}{ EHC Pericos } \\
\cline { 2 - 9 } & $\mathbf{V}$ & C & V & C & V & C & V & C \\
\hline$\underline{r}^{2}$ & 0.82 & $\mathrm{~J}$ & 0.88 & B & 0.81 & $\mathrm{~J}$ & 0.78 & $\mathrm{~J}$ \\
\hline NSE & 0.55 & B & 0.64 & M.B & 0.77 & M.B. & 0.74 & M.B. \\
\hline PBIAS & -125.28 & S.E. & 26.5 & Sub.E. & -57.01 & S.E. & -77.34 & S.E. \\
\hline MAE & 5.2 & N.A. & 11.2 & N.A. & 12.22 & N.A. & 17.55 & N.A. \\
\hline
\end{tabular}

EHC = estación hidrométrica convencional; $\mathrm{V}=$ valor; $\mathrm{C}=$ clasificación; $\mathrm{J}=$ justo; $\mathrm{B}=$ bueno; M.B. = muy bueno; $\mathrm{E}=$ excelente; J = justo; N.A. = no aplica; S.E. = sobreestimación; Sub.E = subestimación. 
Tecnología y

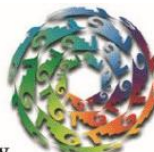

Ciencias đagua
2022, Instituto Mexicano de Tecnología del Agua

Open Access bajo la licencia CC BY-NC-SA 4.0 (https://creativecommons.org/licenses/by-nc$\mathrm{sa} / 4.0 /)$
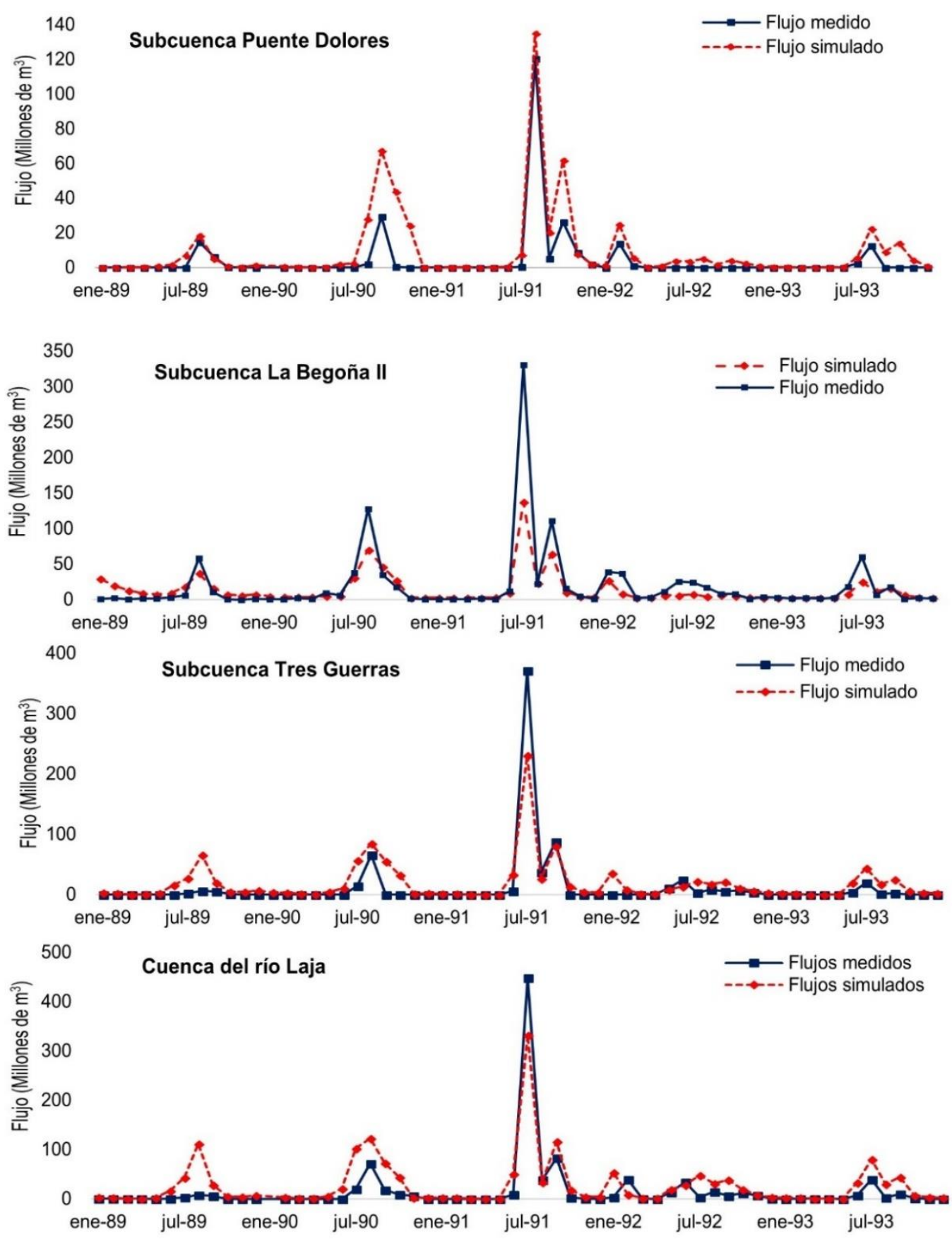

Figura 5. Flujo medido y simulado en el proceso de calibración por WEAP en las subcuencas y cuenca del río Laja. 
Tecnología y

Ciencias $₫$ Agua
2022, Instituto Mexicano de Tecnología del Agua Open Access bajo la licencia CC BY-NC-SA 4.0 (https://creativecommons.org/licenses/by-nc$\mathrm{sa} / 4.0 /)$

Con base en la clasificación de Molnar (2011), valores de NSE menores a 0.2 indica que el ajuste es insuficiente; entre 0.2 y 0.4 es satisfactorio; entre 0.4 y 0.6 es bueno; entre 0.6 y 0.8 es muy bueno; si es mayor a 0.8 es excelente. El coeficiente de determinación fue clasificado con base en Andersen, Refsgaard y Jensen (2001).

De acuerdo con los resultados obtenidos en la calibración se observa que para caudales pequeños el modelo sobrestima; sin embargo, para caudales grandes subestima en todas las subcuencas y a nivel general. La desviación del MAE se incrementó en el orden de localización en el que están las estaciones hidrométricas en la cuenca, presentándose el menor error en la EHC Puente Dolores, localizada en la parte alta de la cuenca, y el mayor en la EHC Pericos, a la salida de la cuenca.

\section{Validación}

De acuerdo con las medidas de bondad de ajuste que se obtuvieron en el proceso de validación, con datos de las EMCs para el periodo de registro de precipitación de 1984-1988 (subcuenca Puente Dolores) y 2001-2005 (La Begoña II, Tres Guerras y Pericos), el coeficiente de determinación obtenido osciló entre 0.65 y 0.86 en las estaciones hidrométricas. El 
Tecnología y

Ciencias $\stackrel{\unlhd}{\unlhd}$ gua
2022, Instituto Mexicano de Tecnología del Agua Open Access bajo la licencia CC BY-NC-SA 4.0 (https://creativecommons.org/licenses/by-nc$\mathrm{sa} / 4.0 /)$

coeficiente de Nash-Sutcliffe en las estaciones hidrométricas es clasificado como muy bueno con base en la clasificación de Molnar (2011), el PBIAS en la mayoría fue negativo e indica sobrestimación del modelo (Tabla 4).

Tabla 4. Medidas de bondad de ajuste para la validación en las subcuencas y cuenca del río Laja.

\begin{tabular}{|c|c|c|c|c|c|c|c|c|}
\hline \multirow[t]{2}{*}{ Prueba } & \multicolumn{2}{|c|}{$\begin{array}{c}\text { EHC Puente } \\
\text { dolores }\end{array}$} & \multicolumn{2}{|c|}{$\frac{\text { EHC Begoña }}{\underline{\text { II }}}$} & \multicolumn{2}{|c|}{$\begin{array}{l}\text { EHC Tres } \\
\text { Guerras }\end{array}$} & \multicolumn{2}{|c|}{$\begin{array}{c}\begin{array}{c}\text { EHC } \\
\text { Pericos }\end{array} \\
\end{array}$} \\
\hline & $\underline{\mathbf{v}}$ & $\underline{\mathrm{C}}$ & $\underline{\mathbf{v}}$ & $\underline{\mathrm{C}}$ & $\underline{\mathbf{v}}$ & $\underline{\mathrm{C}}$ & $\underline{\mathbf{v}}$ & $\underline{\mathrm{C}}$ \\
\hline$\underline{r^{2}}$ & $\underline{0.65}$ & $\underline{P}$ & $\underline{0.86}$ & $\underline{B}$ & $\underline{0.79}$ & $\underline{\mathrm{j}}$ & $\underline{0.71}$ & $\underline{\mathrm{J}}$ \\
\hline NSE & 0.64 & M.B. & 0.62 & M.B. & $\underline{0.75}$ & M.B. & $\underline{0.57}$ & M.B. \\
\hline PBIAS & $\begin{array}{c}= \\
22.23\end{array}$ & S.E. & 26.1 & Sub.E. & $\begin{array}{c}= \\
31.84\end{array}$ & S.E. & $\begin{array}{c}= \\
89.79\end{array}$ & S.E. \\
\hline MAE & 3.67 & N.A. & $\underline{11.0}$ & N.A. & $\underline{10.88}$ & N.A. & $\underline{16.67}$ & N.A. \\
\hline
\end{tabular}

EHC = estación hidrométrica convencional; $\mathrm{V}=$ valor; $\mathrm{C}=$ clasificación; $\mathrm{P}=$ pobre; $\mathrm{J}=$ justo; $\mathrm{B}=$ bueno; M.B. = muy bueno; $\mathrm{E}=$ excelente; N.A. = no aplica; S.E. = sobrestimación; Sub.E. = subestimación.

Los resultados obtenidos en el proceso de validación de las subcuencas y cuenca del río Laja (Figura 6) presentan el mismo comportamiento que en la calibración; de forma general, el modelo sobrestima, no así en flujos pico. 
Tecnología y

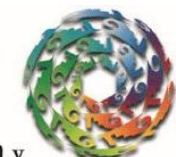

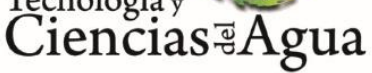

2022, Instituto Mexicano de Tecnología del Agua

Open Access bajo la licencia CC BY-NC-SA 4.0 (https://creativecommons.org/licenses/by-nc$\mathrm{sa} / 4.0 /)$
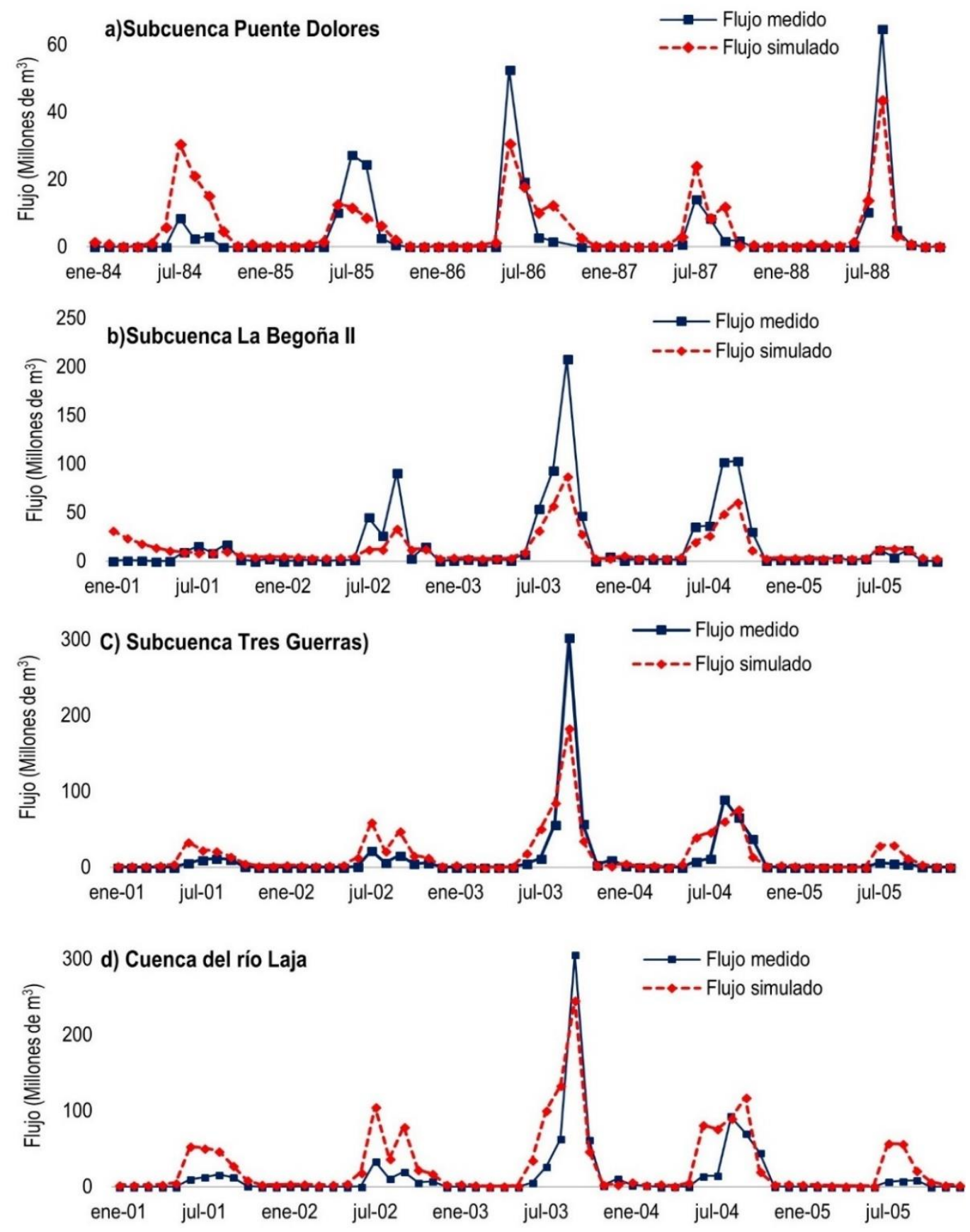

Figura 6. Flujo observado y simulado en el proceso de validación por WEAP en las subcuencas y cuenca del río Laja. 
Tecnología y

Ciencias đgua
2022, Instituto Mexicano de Tecnología del Agua Open Access bajo la licencia CC BY-NC-SA 4.0 (https://creativecommons.org/licenses/by-nc$\mathrm{sa} / 4.0 /)$

\section{Escenario de cambio en clima}

El escurrimiento modelado corresponde a las proyecciones del escenario RCP8.5 Para la cuenca del río Laja se generaron series de tiempo de las variables climáticas correspondiente a temperaturas y precipitación, se corrió el modelo y se observó el comportamiento de los escurrimientos en los puntos de control estudiados.

Para la evaluación del escenario RCP8.5 se utilizó el generador climático WGEN, de tiempo estocástico, desarrollado por el servicio de Investigación Agrícola del Departamento de Agricultura de los Estados Unidos (Richardson \& Wright, 1984). Utiliza estadísticas mensuales y anuales para generar series de tiempo sintéticas diarias de precipitación, temperatura mínima, temperatura máxima y radiación solar; este generador climático está incluido en el módulo Weatherman en el Decision Support System for Agrotechnology Transfer (DSSAT) (Hoogenboom et al., 2017).

La generación climática se realizó para el periodo 2012-2022, basándose en datos de 30 años, con la que se generaron las variables climáticas diarias, correspondientes a precipitación, temperatura mínima, 
Tecnología y

Ciencias $₫$ Agua
2022, Instituto Mexicano de Tecnología del Agua

Open Access bajo la licencia CC BY-NC-SA 4.0 (https://creativecommons.org/licenses/by-nc$\mathrm{sa} / 4.0 /)$

temperatura máxima y radiación solar; posteriormente se realizó el procesamiento necesario para obtener las variables por mes.

La generación del escenario se realizó para cada una de las subcuencas. Con el modelo calibrado se procedió a cambiar la información climática por la generada, con lo cual se obtuvieron los escurrimientos esperados de acuerdo con la precipitación estimada (Figura 7). El escenario RCP8.5 sólo incluye cambio de clima, sin modificar el uso de suelo.

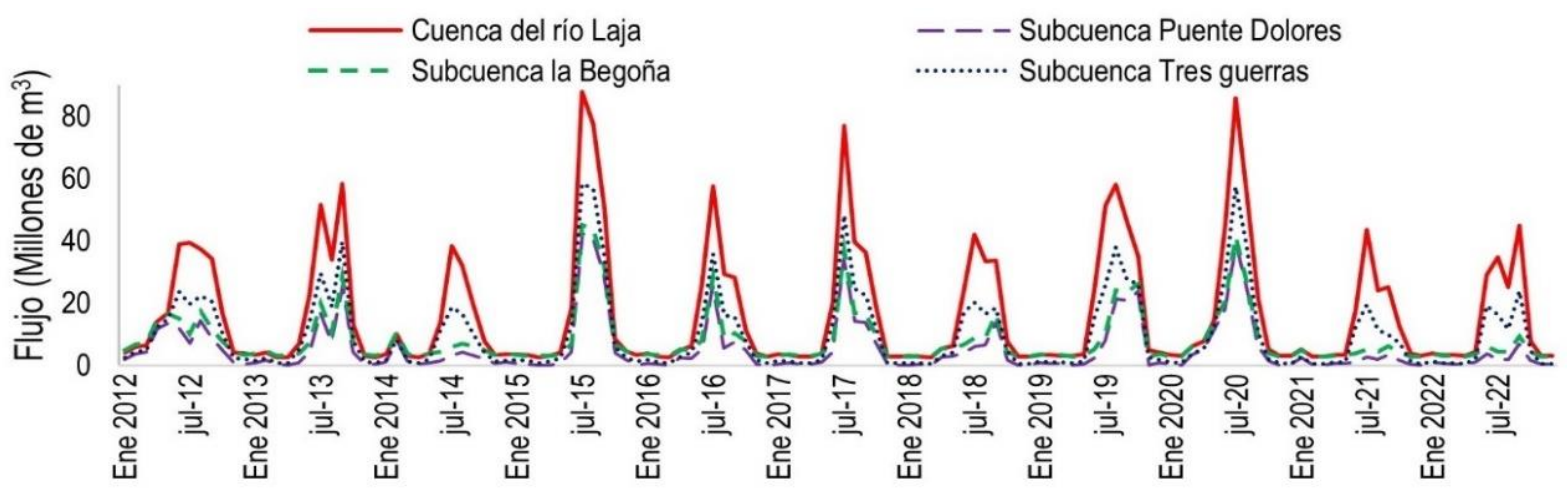

Figura 7. Flujos obtenidos del escenario con cambio de precipitación.

El promedio de los escurrimientos anuales simulados obtenido con las proyecciones del escenario RCP8.5 en la cuenca del río laja es de 184.26 hm³ para el periodo 2012-2022. Palacios-Vélez y López-López (2004) mencionan que la media aritmética de los escurrimientos en la cuenca del río Laja es de $183.982 \mathrm{hm}^{3}$; este dato confirma lo que dicen Bolongaro-Crevenna et al. (2016); el escenario RCP8.5 estima poco cambio en la cantidad de precipitación total. 
Tecnología y

Ciencias $₫$ Agua
2022, Instituto Mexicano de Tecnología del Agua

Open Access bajo la licencia CC BY-NC-SA 4.0 (https://creativecommons.org/licenses/by-nc$\mathrm{sa} / 4.0 /)$

\section{Conclusiones}

Los modelos hidrológicos son una herramienta útil para simular los procesos que ocurren en una cuenca; los resultados que se obtienen de ellos facilitan la toma de decisiones e implementación de políticas públicas para el uso eficiente y aprovechamiento de los recursos hídricos.

El resultado que se obtuvo de la aplicación de la herramienta WEAP en la cuenca del río Laja indica que el método de humedad del suelo es capaz de reproducir satisfactoriamente la respuesta de la cuenca del río Laja con base en los índices de eficiencia NSE, PBIAS y $r^{2}$.

El modelo fue calibrado y validado para un periodo de cinco años en cada una de las subcuencas de la cuenca del río laja. El modelo calcula mayores caudales en comparación con el caudal medido; no sucede lo mismo en los caudales pico, donde WEAP subestima los caudales.

Del presente trabajo es importante mencionar que una de las ventajas de WEAP al ser un modelo conceptual-base física es la cantidad de datos requeridos para su alimentación, que se considera menor en comparación con otros modelos hidrológicos; sin embargo, el rango de 
Tecnología y

Ciencias $₫$ Agua
2022, Instituto Mexicano de Tecnología del Agua Open Access bajo la licencia CC BY-NC-SA 4.0 (https://creativecommons.org/licenses/by-nc$\mathrm{sa} / 4.0 /)$

variación de los valores de los parámetros que integran el modelo no están definidos.

\section{Agradecimientos}

Los autores agradecen al Conacyt y al INIFAP-CENID-RASPA (Dr. Ignacio Sánchez Cohen, M.C. Gerardo Esquivel Arriaga) por su apoyo y colaboración para el desarrollo de la presente investigación.

\section{Referencias}

Ahmed, F. (2012). A hydrologic model of kemptville basin calibration and extended validation. Water Resources Management, 26(9), 25832604. DOI:10.1007/s11269-012-0034-0

Allen, R. G., Pereira, L. S., Raes, D., \& Smith, M. (2006). Estudio FAO riego y drenaje 56. Evapotranspiración del cultivo (guías para la determinación de los requerimientos de agua de los cultivos). Roma, Italia: Food and Agriculture Organization of the United Nations. Recuperado de http://www.fao.org/3/a-x0490s.pdf

Amato, C. C., McKinney, D. C., Ingol-Blanco, E., \& Teasley, R. L. (2006). WEAP hydrology model applied: the Rio Conchos Basin. Center for Research in Water Resources, University of Texas at Austin, USA. Recuperado de https://repositories.lib.utexas.edu/bitstream/handle/2152/7025/crw r_onlinereport06-12.pdf?sequence $=2$

Andersen, J., Refsgaard, J. C., \& Jensen, K. H. (2001). Distributed 
Teçnología y

Ciencias $\stackrel{\unlhd}{\unlhd}$ gua
2022, Instituto Mexicano de Tecnología del Agua Open Access bajo la licencia CC BY-NC-SA 4.0 (https://creativecommons.org/licenses/by-nc$\mathrm{sa} / 4.0 / /)$

hydrological modelling of the Senegal River Basin-model construction and validation. Journal of Hydrology, 247(3-4), 200-214. DOI: 10.1016/S0022-1694(01)00384-5

Bolongaro-Crevenna, A., Márquez-García, A. Z., Torres-Rodríguez, V., Origel-Gutiérrez, G., Márquez-García, E., Aldeco-Ramírez, J., DíazFlores, M. A., Fernández-Bringas, L. M., Gómez Mendoza, L., GalvánOrtiz, L. M., Gómez-Millán, D. G., Ávila-Flores, B., Solares-Hernández, H., Montes-León, M. A. L., Márquez-García, L. I., Márquez-Hernández, A. C., Adriana-Pérez, E., Verónica Pérez-Flores, E. V., García-Díaz, B. E., \& Arzate-Ramírez, S. G. (2016). Diagnóstico de la vulnerabilidad ante el cambio climático del destino turístico de San Miguel de Allende, Guanajuato. En: Bolongaro-Crevenna, R. A. (coord.). Estudio de vulnerabilidad al cambio climático en diez destinos turísticos seleccionados (Informe Técnico Proyecto 238980). Ciudad de México, México: Academia Nacional de Investigación y Desarrollo, Fondo Sectorial para la Investigación en Desarrollo y la Innovación Tecnológica en Turismo Conacyt-Sectur. Recuperado de http://www.sectur.gob.mx/wp-content/uploads/2018/01/6_SanMiguel-de-Allende.pdf

Bonelli, S., Vicuña, S., Meza, F. J., Gironás, J., \& Barton, J. (2014). Incorporating climate change adaptation strategies in urban water supply planning: The case of central Chile. Journal of Water and Climate Change, 5(3), 357-376.DOI: 10.2166/wcc.2014.037

Cabrera, J. (2012). Calibración de modelos hidrológicos. Lima, Perú: Universidad Nacional de Ingeniería Civil, Instituto para la Mitigación 
Tecnología y

Ciencias $\stackrel{\unlhd}{\unlhd}$ gua
2022, Instituto Mexicano de Tecnología del Agua Open Access bajo la licencia CC BY-NC-SA 4.0 (https://creativecommons.org/licenses/by-nc$\mathrm{sa} / 4.0 /)$

de los Efectos del Fenómeno El Niño.

Campos, D. F. (1998). Procesos del ciclo hidrológico. San Luis Potosí, México: Editorial Universitaria Potosina, Universidad Autónoma de San Luis Potosí.

CCG, Centro de Cambio Global. (2009). Guía metodológica: modelación hidrológica y de recursos hídricos con el modelo WEAP. Santiago/Chile, Boston/EUA: Universidad Católica de Chile, Stockholm Environment Institute. Recuperado de http://www.weap21.org/downloads/Guia_modelacion_WEAP_Espano I.pdf

Choi, S. J., Kim, J. H., \& Lee, D. R. (2012). Decision of the water shortage mitigation policy using multi-criteria decision analysis. KSCE Journal of Civil Engineering, 16(2), 247-253. DOI: 10.1007/s12205-0120008-z

Conagua, Comisión Nacional del Agua. (2014). Estadísticas del agua en México. México. Recuperado de http://www.conagua.gob.mx/CONAGUA07/Publicaciones/Publicacion es/EAM2014.pdf

Conagua \& IMTA, Comisión Nacional del Agua \& Instituto Mexicano de Tecnología del Agua. (2008). Banco Nacional de Datos de Aguas Superficiales (BANDAS). Recuperado de

https://app.conagua.gob.mx/bandas/

DOF, Diario Oficial de la Federación. (2003). Acuerdo por el que se dan a conocer las denominaciones y la ubicación geográfica de las 
Tecnología y

Ciencias $₫$ Agua
2022, Instituto Mexicano de Tecnología del Agua Open Access bajo la licencia CC BY-NC-SA 4.0 (https://creativecommons.org/licenses/by-nc$\mathrm{sa} / 4.0 /)$

diecinueve cuencas localizadas en la zona hidrológica denominada río Lerma-Chapala, así como la disponibilidad media anual de las aguas superficiales en las cuencas que comprende dicha zona hidrológica. México, DF, México: Secretaría del Medio Ambiente y Recursos Naturales.

Esquivel, A. G., Nevárez, F. M. M, Velásquez, V. M. A., Sánchez, C. I., \& Bueno, H. P. (2017). Hydrological modeling of a basin in Mexico's arid northern region and its response to environmental changes. Ingeniería Agrícola y Biosistemas, 9(1), 3-18. DOI: 10.5154/r.inagbi.2016.12.008

Febrillet-Huertas, J. F., José-Clases, S., Bello, L., \& Chalas, J. (2014). Simulación de los efectos del cambio climático en los recursos hídricos y estrategias de adaptación, usando el modelo Water Evaluation and Planning (WEAP), en la cuenca de la región Yaque del Norte de la República Dominicana. Aqua-LAC, 6(2), 21-36. DOI: 10.29104/phiaqualac/2014-v6-2-03

Flores-López, F., Galaitsi, S. E., Escobar, M., \& Purkey, D. (2016). Modeling of andean páramo ecosystems' hydrological response to environmental change. Water, 8(3), 94. DOI: 10.3390/w8030094

Hamlat, A., Errih, M., \& Guidoum, A. (2013). Simulation of water resources management scenarios in western Algeria watersheds using WEAP model. Arabian Journal of Geosciences, 6(7), 2225-2236. DOI: $10.1007 / \mathrm{s} 12517-012-0539-0$

Harma, K. J., Johnson, M. S., \& Cohen, S. J. (2012). Future water supply and demand in the Okanagan Basin, British Columbia: A scenario- 
Tecnología y

Ciencias $₫$ Agua
2022, Instituto Mexicano de Tecnología del Agua Open Access bajo la licencia CC BY-NC-SA 4.0 (https://creativecommons.org/licenses/by-nc$\mathrm{sa} / 4.0 /)$

based analysis of multiple, interacting stressors. Water Resources Management, 26(3), 667-689. DOI: 10.1007/s11269-011-9938-3

Höllermann, B., Giertz, S., \& Diekkrüger, B. (2010). Benin 2025Balancing future water availability and demand using the WEAP 'Water Evaluation and Planning' System. Water Resources Management, 24(13), 3591-3613. DOI: 10.1007/s11269-010-9622Z

Hoogenboom, G., Porter, C., Vakhtang, S., Boote, K., Singh, U., White, J., Hunt, L., Ogoshi, R., Lizaso, J. I., Koo, J., Asseng, S., Singels, A., Moreno, L., \& Jones, J. (2017). Decision Support System for Agrotechnology Transfer (DSSAT) Version 4.7 (https://DSSAT.net). Gainesville, USA: DSSAT Foundation.

INEGI, Instituto Nacional de Estadística y Geografía. (2013). Continental

- Continuo de elevaciones mexicano, CEM. Versión 3.0. Aguascalientes, México: Instituto Nacional de Estadística y Geografía. Recuperado de http://www.inegi.org.mx/geo/contenidos/datosrelieve/continental/D escarga.aspx

INEGI, Instituto Nacional de Estadística y Geografía. (2010). Conjunto de datos vectoriales de la carta de uso de suelo y vegetación, escala 1:250 00 Serie IV (Continuo Nacional). Aguascalientes, México: Instituto Nacional de Estadística y Geografía. Recuperado de http://www.inegi.org.mx/geo/contenidos/recnat/usosuelo/Default.as px.

INIFAP, Instituto Nacional de Investigaciones Forestales Agrícolas y 
Tecnología y

Ciencias $₫$ Agua
2022, Instituto Mexicano de Tecnología del Agua Open Access bajo la licencia CC BY-NC-SA 4.0 (https://creativecommons.org/licenses/by-nc$\mathrm{sa} / 4.0 /)$

Pecuarias. (2016). Red Nacional de Estaciones Agrometeorológicas Automatizadas INIFAP. Ciudad de México: Instituto Nacional de Investigaciones Forestales Agrícolas y Pecuarias. Recuperado de http://clima.inifap.gob.mx/redinifap/

Johnston, R., \& Smakhtin, V. (2014). Hydrological modeling of large river Basins: How much is enough? Water Resources Management, 28(10), 2695-2730. DOI: 10.1007/ s11269-014-0637-8

Magaña, V. O. (2005). Fomento de las capacidades para la etapa II de adaptación al cambio climático en Centroamérica, México y Cuba. Estudio de caso en México: Tlaxcala. Análisis de la vulnerabilidad actual, Informe de Avance de Trabajo (Vol. 31) (Proyecto RLA/01/G31/A/1G/99). Programa dee las Naciones Unidas para el Desarrollo, Fondo Global del Ambiente. Recuperado de http://www2.inecc.gob.mx/descargas/cclimatico/cc_cmc_regional.p df

Molnar, P. (2011). Calibration. Watershed modelling, SS 2011. Zürich, Switzerland: Institute of Environmental Engineering, Chair of Hydrology and Water Resources Management, ETH.

Palacios-Vélez, E., \& López-López, C. (2004). La sobreexplotación de las cuencas hidrológicas: el caso de la cuenca del río de La Laja, Guanajuato. En: Cotler, H. (ed.). El manejo integral de cuencas en México (pp. 131-148). México, DF, México: Secretaría de Medio Ambiente y Recursos Naturales, Instituto Nacional de Ecología. Recuperado de http://www2.inecc.gob.mx/publicaciones2/libros/528/sobreexplotaci 
Teçnología y

Ciencias $\stackrel{\mho}{\varpi}$ Aua
2022, Instituto Mexicano de Tecnología del Agua Open Access bajo la licencia CC BY-NC-SA 4.0 (https://creativecommons.org/licenses/by-nc$\mathrm{sa} / 4.0 /)$

on.pdf

Refsgaard, J. C., \& Knudsen, J. (1996). Operational validation and intercomparison of different types of hydrological models. Water Resources Research, 32(7), 2189-2202. DOI: 10.1029/96WR00896

Richardson, C. W., \& Wright, D. A. (1984). WGEN: A model for generating daily weather variables, ARS-8. Washington, DC, USA: US Department of Agriculture, Agricultural Research Service.

Rochdane, S., Reichert, B., Messouli, M., Babqiqi, A., \& Khebiza, M. Y. (2012). Climate change impacts on water supply and demand in Rheraya Watershed (Morocco), with potential adaptation strategies. Water, 4(1), 28-44. DOI: 10.3390/w4010028

Sánchez-Torres-Esqueda, G., Ospina-Noreña, J. E., Gay-García, C., \& Conde, C. (2011). Vulnerability of water resources to climate change scenarios. Impacts on the irrigation districts in the Guayalejo-Tamesí river basin, Tamaulipas, México. Atmósfera, 24(1), 141-155.

Sandoval-Solis, S., \& McKinney, D. C. (2009). Hydrological feasibility of environmental flows in the Río Grande/Bravo basin. In: World Environmental and Water Resources Congress 2009: Great Rivers (pp. 1-11). DOI: 10.1061/41036(342)497

Savage, M., Rodrigo, D., Lund, J., Munevar, A., Purkey, D., \& Bourez, W. (mayo, 2004).Sacramento, California, USA: California Water and Environmental Modeling Forum Technical Workshop, Presentation Summaries, CWEMF.

SMN, Servicio Meteorológico Nacional. (2009). Red de Estaciones 
Tecnología y

Ciencias $₫$ Agua
2022, Instituto Mexicano de Tecnología del Agua Open Access bajo la licencia CC BY-NC-SA 4.0 (https://creativecommons.org/licenses/by-nc$\mathrm{sa} / 4.0 /)$

Climatológicas. Climatología mensual. México, DF, México: Servicio Meteorológico Nacional.

Recuperado de http://smn.cna.gob.mx/index.php?option=com_content\&view=ar ticle\&id $=42 \&$ Itemid $=75$

SEI, Stockholm Environment Institute. (2015). Water evaluation and planning system, WEAP. Boston, USA: Stockholm Environment Institute.

Torres-Benites, E. , Mejía-Sáenz, E., Cortés-Becerra, J., Palacios-Vélez, E., \& Exebio-García, A. (2005). Adaptación de un modelo de simulación hidrológica a la cuenca del río Laja, Guanajuato, México. Agrociencia, 39(5). Recuperado de https://agrocienciacolpos.mx/index.php/agrociencia/article/download/411/411

Yates, D., Miller, K. A., Wilby, R. L., \& Kaatz, L. (2015a). Decision-centric adaptation appraisal for water management across Colorado's continental divide. Climate Risk Management. DOI: 10.1016/j.crm.2015.06.001

Yates, D., Sieber, J., Purkey, D., Huber-Lee, A., \& Galbraith, H. (2005b). WEAP21-A demand, priority, and preference-driven water planning model, part 1: Model characteristics. Water International, 30(4), 487500. DOI: $10.1080 / 02508060508691893$

Yates, D., Sieber, J., Purkey, D., Huber-Lee, A., \& Galbraith, H. (2005c). WEAP21-A demand, priority, and preference driven water planning model, part 2: Aiding freshwater ecosystem service evaluation. Water International, 30(4), 501-512. DOI: 10.1080/02508060508691894 